\title{
Glue Embolization of Gastrobronchial Fistula Post Sleeve Gastrectomy
}

\author{
Abdulmajeed Bin Dahmash ${ }^{1}$ Omar Bashir ${ }^{1}$ Shaima Ahmed Abulqasim ${ }^{1} \quad$ Aljabriyah Alfutais ${ }^{2}$ \\ Mohammad Arabi ${ }^{10}$ Sultan Alhabdan ${ }^{3,4}$ Abdulmohsin Abdulrahman Alzakari ${ }^{3}$ \\ Mohammed Almoaiqel ${ }^{1,4}$
}

1 Department of Medical Imaging, Vascular and Interventional Radiology Unit, King Abdulaziz Medical City \& King Abdullah Specialized Children's Hospital, Ministry of National Guard-Health Affairs, Riyadh, Saudi Arabia

2 Department of Medical Imaging, Vascular and Interventional Radiology Unit, King Abdullah Bin Abdulaziz University Hospital, Princess Nourah Bint Abdulrahman University, Riyadh, Saudi Arabia

${ }^{3}$ Department of Surgery, King Abdulaziz Medical City, Ministry of

National Guard-Health Affairs, Riyadh, Saudi Arabia

${ }^{4}$ King Saud Bin Abdulaziz University for Health Sciences, Riyadh, Saudi Arabia

Arab J Intervent Radiol 2021;5:119-122.

Staple line leak occurs in 1.4 to $2.8 \%$ of cases post sleeve gastrectomy and can be treated by surgical revision, stent placement, or endoscopic interventions. ${ }^{1}$ Untreated, it may progress to gastropleural or gastrobronchial fistulas $(\mathrm{GBF}){ }^{1}$ We report two cases of GBF: one was successfully treated with glue embolization, and the other underwent surgical repair after unsuccessful glue embolization. Institutional research committee at our institute approved this report for publication.

The first case is a 25-year-old female who developed chronic GBF following staple line leak 18 days post sleeve gastrectomy (-Fig. 1). Patient complained of productive cough and shoulder pain. Initial management with a covered gastroesophageal stent for 6 weeks and then a second stenting for 8 weeks failed to treat the fistula. Endoscopic clipping of gastric opening of the fistula also failed. Subsequently, embolization of the fistula was performed via orogastric approach. A 5-F Kumpe catheter (Cook Medical, Indianapolis, Indiana, United States) was used to select the gastric opening of the fistula. Contrast injection confirmed presence of fistula between the stomach and left lower lobe bronchus. Glubran II (GEM Srl, Viareggio, Italy) mixed with lipiodol was injected to successfully embolize the tract ( - Fig. 2). Followup computed tomography (CT) scan at 3 months showed

published online February 7, 2022
DOI https://doi.org/

10.1055/s-0041-1742085. ISSN 2542-7075.
Address for correspondence Omar Bashir, MBBS, FRCR, EBIR, Department of Medical Imaging, Vascular and Interventional Radiology Unit, King Abdulaziz Medical City, Ministry of National Guard-Health Affairs, Riyadh 22490-11426, Kingdom of Saudi Arabia (e-mail: drobashir@gmail.com).

complete resolution of the fistula and marked improvement in the left lower lobe inflammatory changes (-Fig. 3). Clinical follow-up indicated resolution of the patient's symptoms. The second case is a 28-years-old female who also developed GBF post sleeve gastrectomy ( - Fig. 4). Patient's symptoms included cough and hematemesis. A trial of gastroesophageal

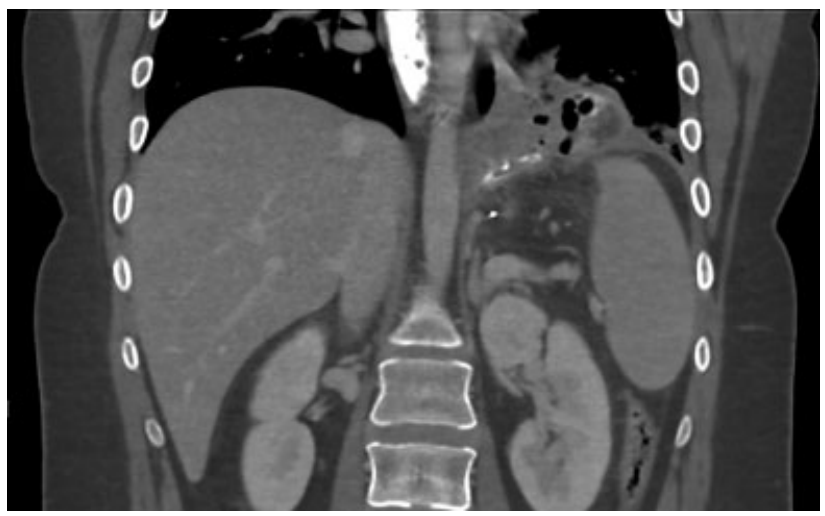

Fig. 1 Coronal-enhanced computed tomographic image showing gastrobronchial fistula between the stomach, left lung, and left lower lobe bronchus. (c) 2022. The Pan Arab Interventional Radiology Society. All rights reserved.

This is an open access article published by Thieme under the terms of the Creative Commons Attribution-NonDerivative-NonCommercial-License, permitting copying and reproduction so long as the original work is given appropriate credit. Contents may not be used for commercial purposes, or adapted, remixed, transformed or built upon. (https://creativecommons.org/ licenses/by-nc-nd/4.0/)

Thieme Medical and Scientific Publishers Pvt. Ltd., A-12, 2nd Floor, Sector 2, Noida-201301 UP, India 


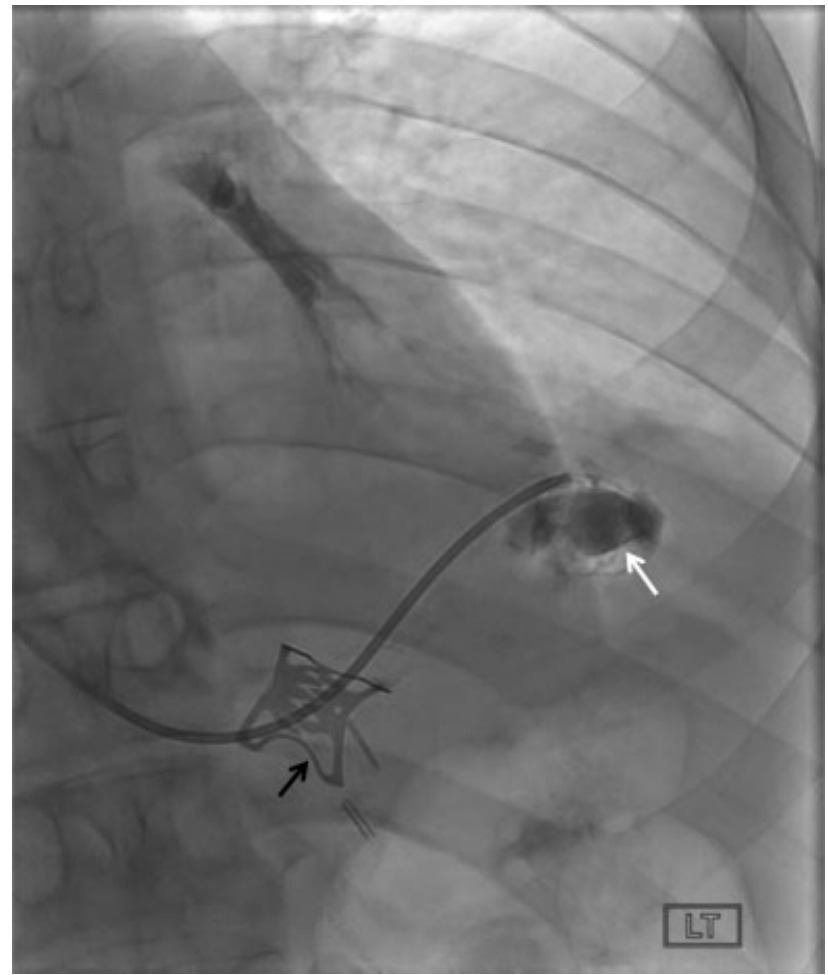

Fig. 2 Intraprocedural fluoroscopy image showing left upper quadrant collection (white arrow) communicating with bronchial tree in left lower lobe via gastrobronchial fistula with successful embolization of the fistula track with Glubran II mixed with lipiodol. Endoscopic clipping also shown in the image (black arrow).

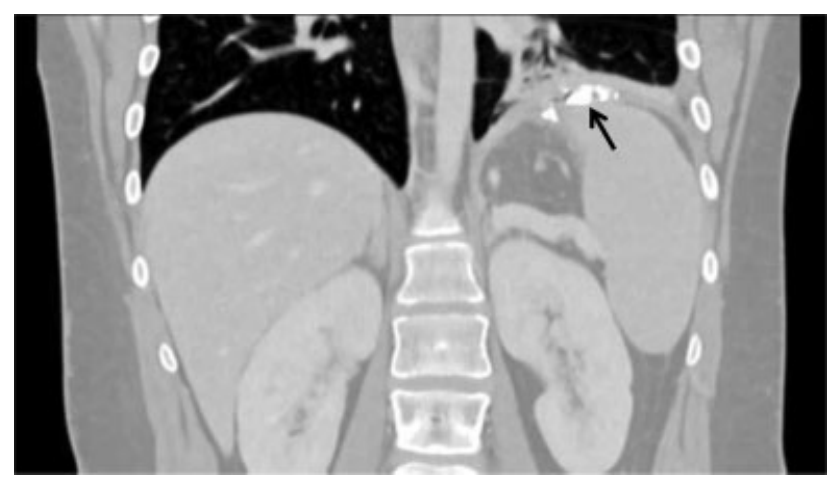

Fig. 3 Coronal-enhanced computed tomographic scan 3 months after the procedure shows successful embolization of the fistula tract with Glubran II (arrow) and improvement in left lower lobe inflammatory changes.

stenting for 13 weeks failed to resolve the fistula. First stent was placed 10 months post-surgery for the duration of 6 weeks and followed by another stent for 7 weeks as the GBF persisted. Patient was refereed to interventional radiology for GBF embolization. Fistula tract was selected with a 5 Fr catheter and microcatheter. Embolization was performed with Glubran II (1:1 Glubran II: Lipiodol) ( - Fig. 5). Follow-up CT scan 1 month later showed persistent GBF associated with unchanged left lower lobe consolidation and plural effusion (-Fig. 6). Patient then underwent successful laparotomy with fistulectomy and Roux-en-Y gastrojejunostomy.

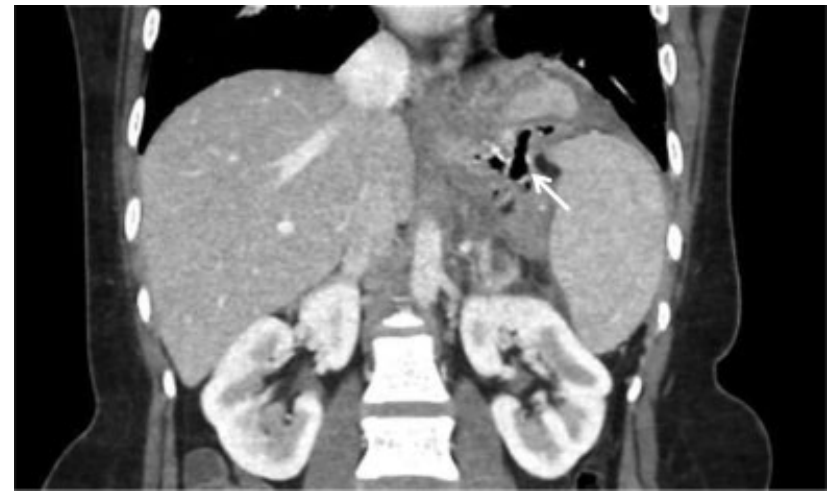

Fig. 4 Coronal-enhanced computed tomographic image showing gastrobronchial fistula track (arrow) arising from the proximal aspect of stomach through the left hemidiaphragm.

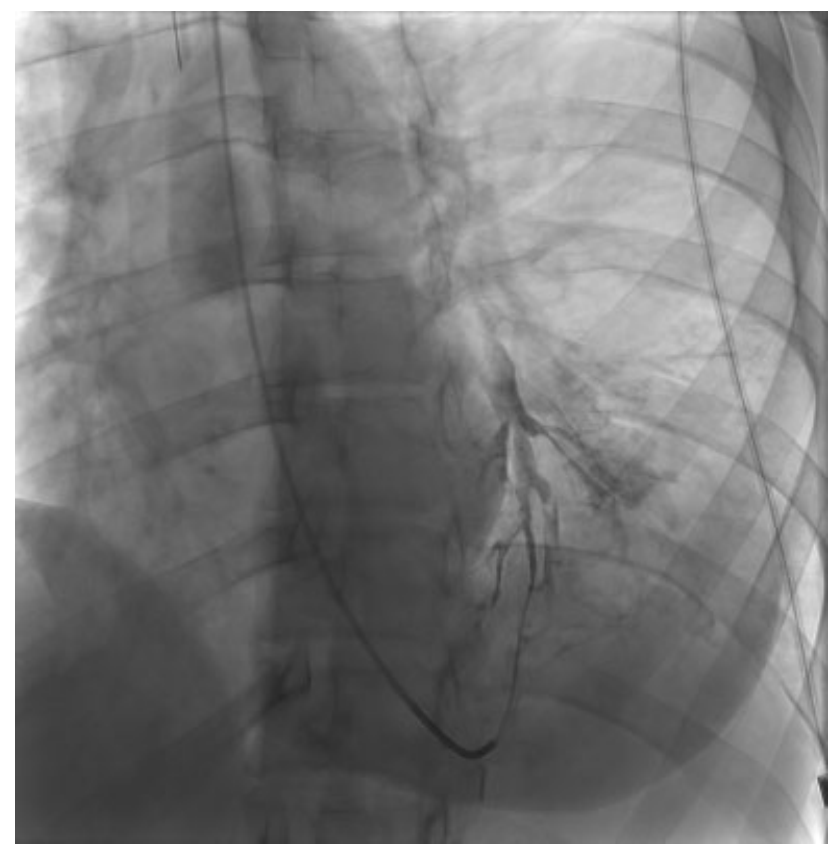

Fig. 5 Intraprocedural fluoroscopy image showing gastrobronchial fistula embolized with Glubran II (1:1 Glubran II: Lipiodol).

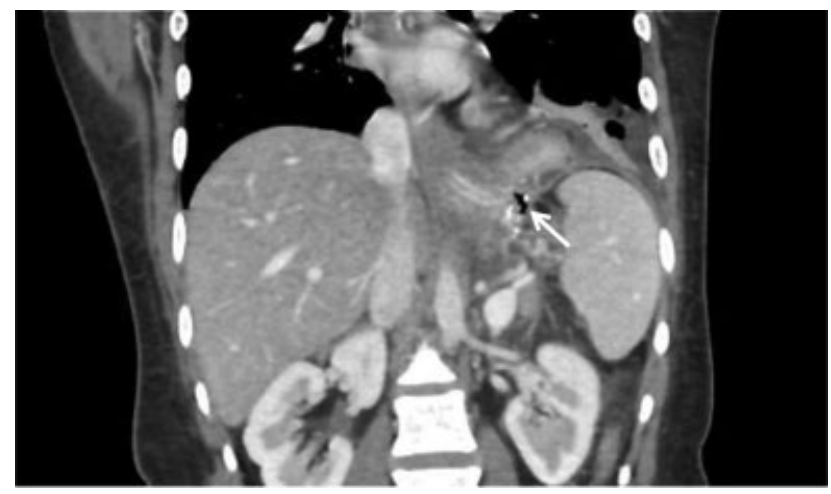

Fig. 6 CT scan 1 month later showing persistent gastrobronchial fistula tract arising from the proximal aspect of stomach through the left hemidiaphragm with the contrast seen in the left lower lobe (arrow). 
The incidence of GBF post sleeve gastrectomy is still unknown ${ }^{1}$ with paucity in medical literature for the best treatment plan for GBF. A retrospective study on 21 patients with GBF treated with gastroesophageal stents showed a success rate of $81 \%{ }^{2}$ Previous case report by Fuks et $\mathrm{al}^{3}$ on a female patient with GBF post sleeve gastrectomy that underwent total gastrectomy with pulmonary lobectomy after failure of several minimally invasive options including stent placement and seven injections of fibrin and Histoacryl glue.

Glue embolization may be considered as a minimally invasive option for the management of GBF post sleeve gastrectomy. Optimization of the technique and proper patient selection are vital for clinical and technical success. However, surgical operation may be required as a definitive intervention when less invasive procedures fail. Further studies are needed to determine the effectiveness of this method.

Conflict of Interest

The authors declare no conflict of interest.

\section{Reference}

1 Guillaud A, Moszkowicz D, Nedelcu M, et al. Gastrobronchial fistula: a serious complication of sleeve gastrectomy. Results of a French multicentric study. Obes Surg 2015;25(12):2352-2359

2 Eisendrath P, Cremer M, Himpens J, Cadière G-B, Le Moine O, Devière J. Endotherapy including temporary stenting of fistulas of the upper gastrointestinal tract after laparoscopic bariatric surgery. Endoscopy 2007;39(07):625-630

3 Fuks D, Dumont F, Berna P, et al. Case report-complex management of a postoperative bronchogastric fistula after laparoscopic sleeve gastrectomy. Obes Surg 2009;19(02):261-264 